\title{
Effect of empagliflozin on albuminuria, eGFR and serum creatinine: updated results from the $A B C D$ nationwide empagliflozin audit
}

\author{
THOMAS SI CRABTREE, ${ }^{1}$ ALEX BICKERTON, ${ }^{2}$ JACKIE ELLIOTT, ${ }^{3}$ RAJEEV RAGHAVAN, ${ }^{4}$ DENNIS BARNES, \\ SIVA SIVAPPRIYAN, ${ }^{6}$ SUZANNE PHILLIPS, 7 ALISON EVANS, ${ }^{7}$ DEVESH SENNIK, ${ }^{8}$ ANURITA ROHILLA, ${ }^{9}$ \\ IAN GALLEN, ${ }^{10}$ ROBERT EJ RYDER, ${ }^{11}$ ABCD EMPAGLIFLOZIN AUDIT CONTRIBUTORS
}

\begin{abstract}
Introduction: Evidence from phase III and the EMPA-REG OUTCOME trials have demonstrated improvements in renal endpoints with empagliflozin use. The EMPA-KIDNEY trial is currently underway and is assessing whether there are benefits of empagliflozin in improving renal outcomes in people both with and without diabetes, and the mechanism has been suggested to be similar to that of ACE inhibitors with the haemodynamic effects of sodium-glucose co-transporter2 inhibition reducing intraglomerular pressure.

Aim: To assess the impacts of empagliflozin use on albuminuria and estimated glomerular filtration rate (eGFR) in a realworld UK-based audit.

Methods: Data were collated via the $A B C D$ nationwide audit programme, with analyses performed using either t-tests/ ANOVA or Wilcoxon signed rank/Kruskal-Wallis tests. Prespecified stratified subgroup analyses by baseline eGFR and baseline albuminuria levels were also performed.

Results: Our results demonstrated significant reductions in albuminuria across the population as a whole. When stratified by baseline albuminuria levels, those with microalbuminuria (30-300 $\mu \mathrm{g} / \mathrm{mg})$ or macroalbuminuria $(>300 \mu \mathrm{g} / \mathrm{mg})$
\end{abstract}

University Hospitals of Derby and Burton NHS Trust; University of Nottingham; Sandwell and West Birmingham Hospitals NHS Trust, UK Yeovil District Hospital NHS Trust, Somerset, UK

${ }^{3}$ Sheffield Teaching Hospitals NHS Foundation Trust, Sheffield, UK

${ }^{4}$ Wolverhampton Diabetes Centre, New Cross Hospital, Wolverhampton, UK

${ }^{5}$ The Tunbridge Wells Hospital, Kent, UK

${ }^{6}$ Maidstone Hospital, Kent, UK

${ }^{7}$ Cheltenham General Hospital, Cheltenham, UK

8 Princess Alexandra Hospital, Harlow, UK

${ }^{9}$ Chief Pharmacist, West Essex CCG, UK

${ }^{10}$ Royal Berkshire NHS Foundation Trust, UK

1 City Hospital, Sandwell and West Birmingham Hospitals NHS Trust, UK

Address for correspondence: Dr Thomas SJ Crabtree Department of Diabetes, Royal Derby Hospital, Uttoxeter Road,

Derby, DE22 3NE, UK

E-mail: t.crabtree@nhs.net

https://doi.org/10.15277/bjd.2021.288 had significant improvements in urine albumin levels at 6month (3-9-month) follow-up, with median changes of -17.7 $\mu \mathrm{g} / \mathrm{mg}(\mathrm{p}<0.0001 ; 95 \% \mathrm{Cl}-17.4$ to -23.7$)$ and $379.4 \mu \mathrm{g} / \mathrm{mg}$ $(p=0.03 ; 95 \% \mathrm{Cl}-269.9$ to -725.4$)$, respectively. Across the population as a whole, eGFR reduced initially (at 6 months, $-1.26 \mathrm{~mL} / \mathrm{min} / 1.73 \mathrm{m3} ; \mathrm{p}<0.0001 ; 95 \% \mathrm{Cl}-0.87$ to -1.64 ) before recovering to baseline by 24 months. When stratified by baseline eGFR, those with reduced renal function (eGFR $<90)$ recovered quickest, with improvements in eGFR noted from baseline by 24 months.

Conclusion: In this real-world analysis, the results are comparable to those in randomised controlled trials and are likely more generalisable to UK clinical practice. Unfortunately, we do not have clinical endpoints such as end-stage renal failure, renal death or dialysis as part of our dataset. Future audits could consider including these data to establish clinical as well as biochemical outcomes.

Br J Diabetes 2021;21:62-66

Key words: empagliflozin, real-world, urinary albumin, albuminuria, renal, eGFR

\section{Introduction}

Following the launch of the Association of British Clinical Diabetologists $(A B C D)$ audit programmes for dapagliflozin and canagliflozin, the $A B C D$ nationwide empagliflozin audit was launched in March 2017. This audit programme has collated more than 9,000 sets of routine clinical data from patients in order to investigate the real-world effects of empagliflozin in a UK-based diabetes clinic population.

Further to the initial launch, a recent expansion to include anonymised datasets from Clinical Commissioning Groups (CCGs), the primary care groups responsible for commissioning care in the UK, has vastly increased the number of patients included, as well as allowing the inclusion of new data to expand analyses or look at some aspects in more detail. The recent addition of albuminuria concentrations has facilitated this analysis.

The effect of empagliflozin on albuminuria has been well documented. The initial phase III trials showed improvements in 
albuminuria and, whilst there was a reduction in the estimated glomerular filtration rate (eGFR) on commencement, further decreases appeared to be less than with placebo. ${ }^{1}$ Subsequent to phase III trials, sustained reductions in albuminuria were noted in the EMPA-REG OUTCOME trial, with great reductions observed in those with microalbuminuria $(-25 \%, 95 \% \mathrm{Cl}-31 \%$ to $-19 \%, p<0.0001)$ and macroalbuminuria $(-32 \%, 95 \% \mathrm{Cl}$ $-41 \%$ to $-23 \%, p<0.0001$ ) occurring within 12 weeks of commencement and sustained for the duration of treatment. ${ }^{2}$ Furthermore, results from the EMPA-REG OUTCOME trial showed initial reductions in eGFR with a subsequent reduction in the slope of eGFR decrease thereafter, suggesting a renoprotective effect. ${ }^{3}$ Clinical renal outcomes such as need for dialysis or renal death were also assessed and the risk has been shown to be reduced. Importantly, the outcomes above were mostly independent of changes in $\mathrm{HbA}_{1 \mathrm{c}}$, weight or blood pressure. ${ }^{4}$

The above evidence has led to the establishment of the EMPA-KIDNEY trial to look at the renal outcomes in people both with and without diabetes, which commenced in January 2019 and is expected to complete in October 2022. ${ }^{4}$

There are several proposed mechanisms of action which include the reduction of blood glucose and weight, thus protecting the renal glomerulus from the effects of hyperglycaemia. ${ }^{5}$ Additionally, sodium-glucose co-transporter-2 (SGLT2) inhibitors have been shown to cause haemodynamic effects due to of their effects in causing a naturesis which results in actions similar to ACE inhibition. 5,6 There is some evidence in animal models that SGLT2 inhibitors may downregulate the renin-angiotension system activation caused by hyperglycaemia. ${ }^{7}$ Although empagliflozin causes an initial decrease in eGFR, it provides protection in the long term and has been demonstrated to reduced urinary albumin-creatinine ratios in those with micro- or macroalbuminuria at baseline. ${ }^{2}$

The aim of this observation study is to assess changes in eGFR and albuminuria concentrations following commencement of empagliflozin in a UK-based diabetes population.

\section{Methods}

Data were extracted from the ABCD nationwide audit tool and combined with the anonymised data provided by Eclipse using CCGs registered with the audit programme. Eclipse is a piece of electronic prescription auditing software that is used in clinical practice in some areas of the UK. All datasets including both baseline and follow-up measurements for each variable were included in the analysis. Variables to be assessed in this analysis include serum creatinine $(\mu \mathrm{mol} / \mathrm{L}), \mathrm{eGFR}\left(\mathrm{mL} / \mathrm{min} / 1.73 \mathrm{~m}^{3}\right)$ and albuminuria $(\mu \mathrm{g} / \mathrm{mg})$ at follow-up intervals of 6,12 and 24 months after commencement.

As well as assessing an observed effect across the population as a whole, two sub-analyses were performed to establish whether individuals with differing baseline albuminuria or eGFR measurements exhibited different responses. Well recognised stratified groupings ${ }^{8}$ were used for this as follows:

\section{Albuminuria}

- Albuminuria $<30 \mu \mathrm{g} / \mathrm{mg}$ - normoalbuminuric - Group 1
- Albuminuria 30-300 $\mu \mathrm{g} / \mathrm{mg}$ - microalbuminuric range Group 2

- Albuminuria $>300 \mu \mathrm{g} / \mathrm{mg}$ - macroalbuminuric range Group 3

\section{eGFR}

- eGFR $>90 \mathrm{~mL} / \mathrm{min} / 1.73 \mathrm{~m}^{3}-$ CKD1

- eGFR $60-90 \mathrm{~mL} / \mathrm{min} / 1.73 \mathrm{~m}^{3}$ - CKD2

- eGFR 45-59 mL/min/1.73 m³ - CKD3a

- eGFR $<45 \mathrm{~mL} / \mathrm{min} / 1.73 \mathrm{~m}^{3}$ - CKD3+ (note: this would be offlicence use of empagliflozin, numbers expected to be small so CKD3b-5 grouped together)

Although eGFR values were provided for most patients, many values were capped at 60 or 90 depending on local reporting protocols. In order to ensure the data analysed were as consistent as possible, all eGFR values were recalculated using the Modification of Diet in Renal Disease (MDRD) eGFR equation. ${ }^{9}$ Other equations such as the Chronic Kidney Disease Epidemiology Collaboration (CKD-EPI) equation could not be used due to lack of necessary information such as ethnicity. ${ }^{10}$

\section{Statistical analysis and data handling}

Data were screened for possible erroneous, outlying or duplicate results. Statistical analysis was performed using StataSE 16. Data were assessed for skewness. Variables not following a normal distribution on assessment of skew were analysed using nonparametric methods such as the Wilcoxon signed rank test or Kruskal-Wallis test. All variables following a normal distribution were analysed using t-tests, ANOVA and regression.

\section{Results}

Initially, 9,142 datasets were extracted from the online audit tool and from the data provided from seven CCGs. These data were reviewed and extreme, erroneous or duplicate entries were removed. A total of 7,840 had sufficient data for baseline and follow-up (ie, baseline and follow-up for eGFR and albuminuria) to be included in the final analysis.

The baseline characteristics are summarised in Table 1. The mean age of the observed population was $60.6\left(S D_{ \pm}+10.4\right)$ years at baseline with a median duration of diabetes of 8.4 years (IQR 4.9-13.2). The mean baseline $\mathrm{HbA}_{1 c}$ was $9.2 \%(\mathrm{SD} \pm 1.5)$ or $72.2 \mathrm{mmol} / \mathrm{mol}(S D \pm 15.8)$. The average weight at baseline was $98.1 \mathrm{~kg}(\mathrm{SD} \pm 20.1)$ with a BMl of $33.9 \mathrm{~kg} / \mathrm{m}^{2}(\mathrm{SD} \pm 6.6)$. Mean eGFR across the population was $94.2 \mathrm{ml} / \mathrm{min} / 1.73 \mathrm{~m}^{3}(\mathrm{SD} \pm 20.9)$ Baseline mean albuminuria across the population was excessively skewed due to the high proportion $(n=7,281)$ with "normal" results; therefore averages were assessed using median measurements for the microalbuminuric group and macroalbuminuric groups were $50.9 \mu \mathrm{g} / \mathrm{mg}$ (IQR 36.7-89.2) and $562.7 \mu \mathrm{g} / \mathrm{mg}$ (IQR 400.2-693.6) respectively.

At approximately 6 months (3-9 months) following commencement of empagliflozin, a minor change in mean eGFR of $-2.2 \mathrm{~mL} / \mathrm{min} / 1.73 \mathrm{~m}^{3}$ ( $p<0.0001 ; 95 \% \mathrm{Cl}-1.8$ to -2.6$)$ was noted across the population. This initial decrease was stable at 12 months (9-15 months) and by 24 months (18-30 months) the mean eGFR across the population had improved and was no longer statistically significantly different from baseline, with 
Table 1 Baseline characteristics of observed population

\begin{tabular}{|c|c|}
\hline Characteristic & $\mathrm{n}=7,840$ \\
\hline Mean \pm SD age, years & $60.6 \pm 10.4$ \\
\hline Male, \% & 63.5 \\
\hline Median (IQR) diabetes duration, years & $8.4(4.9-13.2)$ \\
\hline Mean \pm SD $\mathrm{HbA}_{1 \mathrm{c}} \%$ & $9.2 \pm 1.5$ \\
\hline Mean \pm SD $\mathrm{HbA}_{1 \mathrm{c}}, \mathrm{mmol} / \mathrm{mol}$ & $71.7 \pm 15.8$ \\
\hline Mean \pm SD BMI, kg/m² & $33.9 \pm 6.6$ \\
\hline Mean \pm SD weight, kg & $98.1 \pm 20.1$ \\
\hline Mean \pm SD serum creatinine, $\mu \mathrm{mol} / \mathrm{L}$ & $73.8 \pm 15.8$ \\
\hline Mean \pm SD eGFR, $\mathrm{mL} / \mathrm{min} / 1.73 \mathrm{~m}^{2}$ & $94.2 \pm 20.9$ \\
\hline Mean \pm SD systolic $B P, \mathrm{mmHg}$ & $133.2 \pm 13.7$ \\
\hline Mean \pm SD diastolic $B P, \mathrm{mmHg}$ & $78.0 \pm 8.9$ \\
\hline \multicolumn{2}{|l|}{ Median (IQR) albuminuria, $\mu \mathrm{g} / \mathrm{mg}$, by group } \\
\hline Normal $-<30 \mu \mathrm{g} / \mathrm{mg}(\mathrm{n}=5,690)$ & $2(0.8-4.4)$ \\
\hline Microalbuminuria $-30-300 \mu \mathrm{g} / \mathrm{mg}(\mathrm{n}=367)$ & $50.9(36.7-89.2)$ \\
\hline Macroalbuminuria $->300 \mu \mathrm{g} / \mathrm{mg}(\mathrm{n}=30)$ & $562.7(400.2-693.6)$ \\
\hline
\end{tabular}

$\mathrm{BMI}$, body mass index; eGFR, estimated glomerular filtration rate; $\mathrm{BP}$, blood pressure; IQR, interquartile range; SD, standard deviation

mean changes of $-0.72 \mathrm{~mL} / \mathrm{min} / 1.73 \mathrm{~m}^{3}(\mathrm{p}=0.83 ; 95 \% \mathrm{Cl}+0.6$ to -0.8$)$ observed.

When stratified by albuminuria at baseline, those with macroalbuminuria exhibited a trend, although not significant, towards decreases in mean eGFR at 6 months of -5.3 $\mathrm{mL} / \mathrm{min} / 1.73 \mathrm{~m}^{3}(\mathrm{p}<0.09,95 \% \mathrm{Cl}+0.96$ to -11.6$)$ which persisted at 24 months $\left(-6.6 \mathrm{~mL} / \mathrm{min} / 1.73 \mathrm{~m}^{3} ; \mathrm{p}=0.06 ; 95 \% \mathrm{Cl}\right.$ +0.30 to -13.5 ), although failing to reach statistical significance throughout. The normoalbuminuric group more or less mirrors the population pattern (mostly due to the numbers in this group). The microalbuminuria group $(30-300 \mu \mathrm{g} / \mathrm{mg}$ ) showed an initial drop in eGFR, statistically significant by 12 months of $-2.63 \mathrm{~mL} / \mathrm{min} / 1.73 \mathrm{~m}^{3}$ ( $\mathrm{p}=0.01 ; 95 \% \mathrm{Cl} 0.62$ to 4.62$)$ with no recovery observed by 24 months.

When stratified by eGFR at baseline, those with reduced renal function demonstrated improvements in eGFR at followup. Very few patients were using empagliflozin with an eGFR $<45 \mathrm{~mL} / \mathrm{min} / 1.73 \mathrm{~m}^{3}(\mathrm{n}=9)$ so, in spite of numerically large improvements in eGFR in this group, the results failed to reach statistical significance.

The results for the effect of empagliflozin on eGFR, both in the population as a whole and in each subgroup, are shown in Figure $1 a$ and $b$.

Following commencement of empagliflozin, across the population as a whole only very small median reductions in albuminuria were noted of $0.2 \mu \mathrm{g} / \mathrm{mg}(\mathrm{p}<0.0001 ; 95 \% \mathrm{Cl}-0.1$ to -0.2 ) at 6 months with a similar result at 12 and 24 months. This is difficult to interpret due to the vast number of patients with normal albuminuria levels, with $75 \%$ of the population having an absolute value of $<6.1 \mu \mathrm{g} / \mathrm{mg}$.

The changes in albuminuria become more apparent when stratified by baseline albuminuria levels. In the normoalbuminuric group, no significant change was noted at follow-up at 6, 12 or 24 months.

In the microalbuminuric group median levels of albuminuria changed by $17.7 \mu \mathrm{g} / \mathrm{mg}(\mathrm{p}<0.0001 ; 95 \% \mathrm{Cl}-17.4$ to -23.7$)$ at 6 months and by $-24.3 \mu \mathrm{g} / \mathrm{mg}(\mathrm{p}<0.0001 ; 95 \% \mathrm{Cl}-18.9$ to -29.7) at 12 months. Changes at 24 months in this group were not statistically significant due to lower numbers at follow-up.

In those with macroalbuminuria (>300 $\mu \mathrm{g} / \mathrm{mg}$ ) at baseline, median albuminuria decreased significantly with a median

Figure 1. (a) Line chart showing the mean estimated glomerular filtration rate (eGFR) at baseline, 6,12 and 24 months following commencement of empagliflozin across the entire population (total $n=7,840$ ) as well as stratified subgroups using baseline albuminuria. (b) Bar chart showing the change in eGFR from baseline at 6, 12 and 24 months following commencement of empagliflozin across the entire population (total $n=7,480$ ) as well as stratified subgroups based on baseline eGFR

(a)

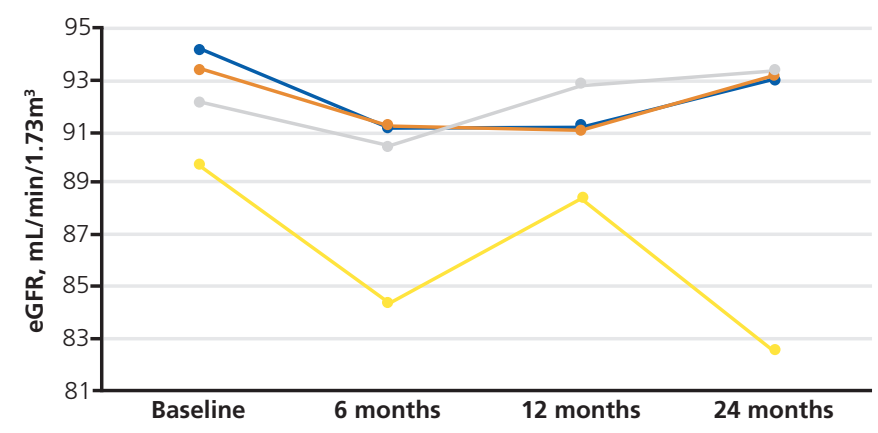

$p<0.05$ for change from baseline if highlighted in red

\begin{tabular}{|lllll|}
\hline $\mathrm{n}=$ & Baseline & 6 months & 12 months & 24 months \\
\hline Normoalbuminuria & 7,281 & 4,209 & 4,067 & 1,463 \\
\hline Microalbuminuria & 511 & 309 & 202 & 77 \\
\hline Macroalbuminuria & 48 & 22 & 25 & 13 \\
\hline Total & 7,840 & 4,540 & 4,294 & 1,553 \\
\hline
\end{tabular}

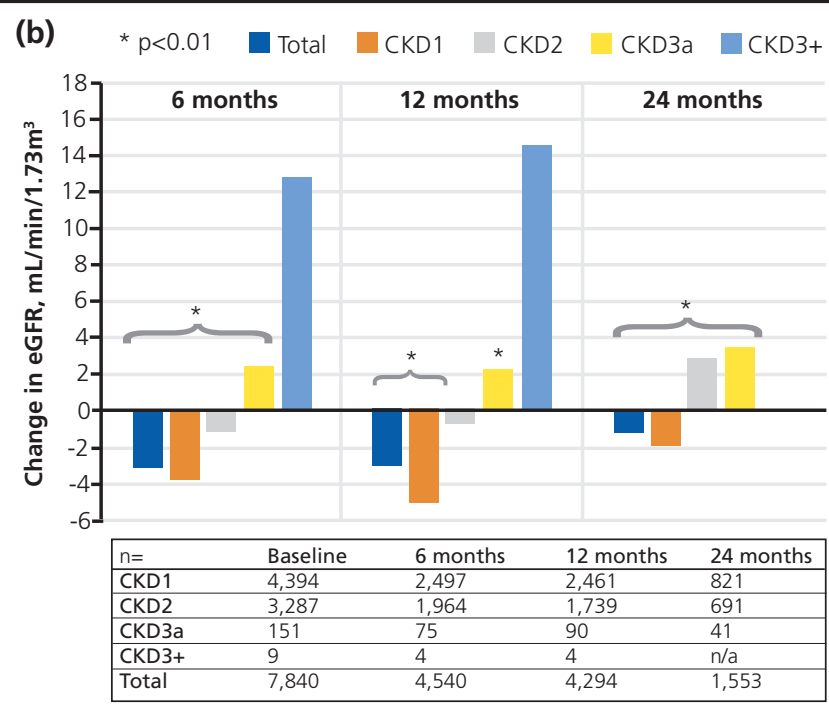


change from baseline of $-379.4 \mu \mathrm{g} / \mathrm{mg}(\mathrm{p}=0.03 ; 95 \% \mathrm{Cl}-269.9$ to -725.4$)$ at 6 months and $-308.8 \mu \mathrm{g} / \mathrm{mg}(\mathrm{p}=0.03 ; 95 \% \mathrm{Cl}$ -116.1 to -637.0 ), although these should be viewed cautiously due to the relatively high $p$ value in the context of multiplicity of analyses. Changes at 24 months were not statistically significant due to lower numbers at follow-up.

When stratified by eGFR, those in groups CKD1 and CKD2 had small statistically significant reductions from baseline at 6 , 12 and 24 months, mirroring the population as a whole. In those with an eGFR of $<60 \mathrm{~mL} / \mathrm{min} / 1.73 \mathrm{~m}^{3}$ in groups CKD3a and $C K D 3 b+$ results failed to reach statistical significance for difference in albuminuria, although this might be due to the much smaller sample sizes in the groups.

Regression analysis demonstrated that reductions in systolic blood pressure correlated significantly with reductions in microalbuminuria across the population as a whole at 6 months $(r=0.48$, $\mathrm{p}=0.01$ ) but not at 12 or 24 months. Changes in other variables including weight, $\mathrm{BMI}$ and $\mathrm{HbA}_{1 \mathrm{c}}$ did not correlate with changes in albuminuria at any time point.

eGFR changes correlated with changes in weight, which reflects the effect that weight may have on increasing creatinine purely due to body mass. They also correlated weakly, although significantly, with systolic blood pressure reductions at 12 and 24 months with coefficients of $0.08(p=0.001)$ and 0.11 $(p=0.01)$, respectively, suggesting decreases in eGFR caused, over this observed period, a further small reduction in eGFR. Baseline albuminuria levels predicted reduction in albuminuria at followup ( $r=0.19, p<0.001$ at 6 months), but no other baseline characteristics correlated.

\section{Discussion}

\section{Key results}

Empagliflozin use is associated with initial decreases in eGFR across the population as a whole at early follow-up (6-12 months), which trends towards baseline values and eventual improvement over a 24-month period in this study. When stratified by baseline eGFR, those with lower values at baseline tended to have more significant early improvements in eGFR, observed as early as 6 months following commencement of treatment. Those with the most preserved renal function at baseline (group CKD1) had reduced eGFR from baseline after 6 months which did not recover completely to baseline, with a statistically significant albeit small reduction in eGFR still present 24 months following commencement.

Albuminuria levels decreased in the population as a whole by a small amount, although the vast majority of this cohort had normal levels of albuminuria at baseline. Those with elevated albuminuria levels at baseline in either the microalbuminuric or macroalbuminuric group appeared to have significant decreases in albuminuria levels associated with empagliflozin use. Although the reduction in urinary albumin for the macroalbuminuric group was large, the significance must be interpreted cautiously due to multiplicity of analyses as $p=0.03$. However, as there are relatively small numbers, it is anticipated that this $p$ value would increase in significance with a larger sample size if it were available.
Reductions in blood pressure correlated weakly although significantly with reduction in eGFR. As commented on in papers on randomised controlled trials, ${ }^{6}$ this might represent an ACE inhibitor-like effect where reduction in blood pressure decreases capsular pressure and therefore reduces urine albumin leakage but, additionally, causes an initial reduction in eGFR. Reductions in weight correlated with increases in eGFR; this could be explained by a decrease in body mass leading to decreases in serum creatinine. Future analyses might include creatinine clearance as a measure in order to incorporate this changing weight into the eGFR equation.

\section{Interpretation}

Our results are comparable with those of the clinical trials discussed in the introduction. The baseline characteristics of our population are more generalisable to a UK-based clinic population as our population had higher levels of baseline $\mathrm{HbA}_{1 \mathrm{c}}$ (eg, $9.2 \%$ vs $8.1 \%$ in EMPA-REG OUTCOME), weighed more (eg, $98.1 \mathrm{~kg}$ vs $86.4 \mathrm{~kg}$ ) and were closer to $50: 50$ male:female participants (eg, $63.5 \%$ vs $72 \%$ male in EMPA-REG OUTCOME). ${ }^{2}$

Our results closely reflected those already reported. Initial drops in eGFR were seen, although recovery and improvements were noted in those with the worst renal functions at baseline (groups CKD2, CKD3a). Albuminuria decreases were noted across the population as a whole and, similar to the randomised controlled trial data, these reductions were most profound with micro- or macroalbuminuria at baseline.

\section{Limitations}

The randomised controlled trials on renal outcomes from empagliflozin and other SGLT2 inhibitors incorporate clinical endpoints into the analyses which we are not able to do with this observation dataset. Future $A B C D$ audit programmes could consider including these as outcomes. Unfortunately, due to the nature of the data collected, no information on discontinuation or adverse effects was available for analysis. Some confounders such as smoking, drinking status, changes to non-diabetes medications or compliance could not be adjusted for in this analysis.

The main effects of SGLT2 inhibition are often observed at 24 months and, unfortunately, we have a paucity of data at this timeframe compared with 6 and 12 months.

\section{Generalisability}

The baseline characteristics of this cohort reflect the general adult diabetes clinic population of the UK fairly well. The inclusion of those with 'off-licence' use and extreme values means our results are likely to be more generalisable to an average clinic population and more reflective of routine clinical practice.

\section{Conclusion}

In this analysis of the $A B C D$ audit programme data, empagliflozin use was associated with decreases in albuminuria levels in those with elevated levels at baseline. Across the population as a whole, eGFR showed initial decreases followed by a return to baseline. Those with worse renal function at base- 


- Key messages
with reductions in urinary albumin creatinine ratios in
those with elevated levels at baseline
Those with preserved renal function at baseline had
associated drops in eGFR following commencement of
Empagliflozin
Conversely, those with eGFR<60ml/min/1.73m² had
improvement in eGFR from baseline by month 24 of
follow-up

line (groups CKD2-CKD3b+) experienced earlier improvements in eGFR, while those with the most preserved renal function (group CKD1) had initial decreases which did not recover completely back to baseline during the 24-month follow-up period. Reductions in systolic blood pressure correlated with reductions in albuminuria and eGFR, and this may be suggestive of a mechanism similar to ACE inhibitors to explain the renal effects of empagliflozin found in this study.

Conflict of interest TSJC reports grants and personal fees from NovoNordisk, grants from Sanofi, outside the submitted work. REJR has received speaker fees, and/or consultancy fees and/or educational sponsorships from AstraZeneca, BioQuest, GI Dynamics, Janssen and Novo Nordisk. $\mathrm{JE}$ and her research department have received educational speaker fees, and/or advisory board fees, from Abbott, DEXCOM, DTN UK, Insulet, Lilly, NovoNordisk, and Sanofi. RR declares payments either as honoraria or speaker fees or towards funding for educational events form the following Eli Lilly and Company, AstraZeneca Ltd, SBK Healthcare Limited, ABCD Ltd, Boehringer Ingelheim, Diabetes UK Ltd, NAPP Pharmaceuticals, Novo Nordisk Ltd. SS declares receipt of speaker fees for the meetings on empagliflozin but not directly related to the effect reported on the effect described in this manuscript. DS reports honoraria for speaker fees paid directly from Boehringer Ingelheim, AstraZeneca, Napp, Sanofi, Novo Nordisk. AB, SP, $A E, I G, A R$ and $D B$ have nothing to declare.
Funding This project is funded by the Association of British Clinical Diabetologists and an unrestricted grant from Boehringer Ingelheim

Acknowledgement: The authors would like to thank the audit contributors (see Appendix 1 at www.bjd-abcd.com)

\section{References}

1. Cherney D, Lund SS, Perkins BA, et al. The effect of sodium glucose cotransporter 2 inhibition with empagliflozin on microalbuminuria and macroalbuminuria in patients with type 2 diabetes. Diabetologia 2016; 59(9):1860-70. https://doi.org/10.1007/s00125-016-4008-2

2. Cherney DZI, Zinman B, Inzucchi SE, et al. Effects of empagliflozin on the urinary albumin-to-creatinine ratio in patients with type 2 diabetes and established cardiovascular disease: an exploratory analysis from the EMPA-REG OUTCOME randomised, placebo-controlled trial. Lancet Diabetes Endocrinol 2017;5(8):610-21. https://doi.org/10.1016/S22138587(17)30182-1

3. Wanner C, Heerspink HJL, Zinman B, et al. Empagliflozin and kidney function decline in patients with type 2 diabetes: a slope analysis from the EMPA-REG OUTCOME trial. J Am Soc Nephrol 2018;29(11):2755-69. https://doi.org/10.1681/ASN.2018010103

4. Herrington WG, Preiss D, Haynes R, et al. The potential for improving cardio-renal outcomes by sodium-glucose co-transporter-2 inhibition in people with chronic kidney disease: a rationale for the EMPA-KIDNEY study. Clin Kidney J 2018;11(6):749-61. https://doi.org/10.1093/ckj/sfy090

5. Burns KD, Cherney D. Renal angiotensinogen and sodium-glucose cotransporter-2 inhibition: insights from experimental diabetic kidney disease. Am J Nephro/ 2019;49(4):328-30. https://doi.org/10.1159/000499598

6. Perrone-Filardi P, Avogaro A, Bonora E, et al. Mechanisms linking empagliflozin to cardiovascular and renal protection. Int J Cardiol 2017; 241:450-6. https://doi.org/10.1016/j.ijcard.2017.03.089

7. Woods TC, Satou R, Miyata K, et al. Canagliflozin prevents intrarenal angiotensinogen augmentation and mitigates kidney injury and hypertension in mouse model of type 2 diabetes mellitus. Am J Nephrol 2019;49(4):33142. https://doi.org/10.1159/000499597

8. Heerspink HJL, Brantsma AH, de Zeeuw D, et al. Albuminuria assessed from first-morning-void urine samples versus 24-hour urine collections as a predictor of cardiovascular morbidity and mortality. Am J Epidemiol 2008; 168(8):897-905. https://doi.org/10.1093/aje/kwn209

9. Levey AS, Bosch JP, Lewis JB, et al. A more accurate method to estimate glomerular filtration rate from serum creatinine: a new prediction equation. Modification of Diet in Renal Disease Study Group. Ann Intern Med 1999; 130(6):461-70. https://doi.org/10.7326/0003-4819-130-6-19990316000002

10. Levey AS, Stevens LA, Schmid $\mathrm{CH}$, et al. A new equation to estimate glomerular filtration rate. Ann Intern Med 2009;150(9):604-12. https://doi.org/10.7326/0003-4819-150-9-200905050-00006 
Appendix 1. ABCD nationwide and worldwide empagliflozin audit contributors The following are those whom we know about

$A B C D$ nationwide empagliflozin audit - initial setup, maintenance and nationwide analysis:

Ryder REJ, Crabtree T, Cull ML, Yadagiri M. Statistician: Blann A

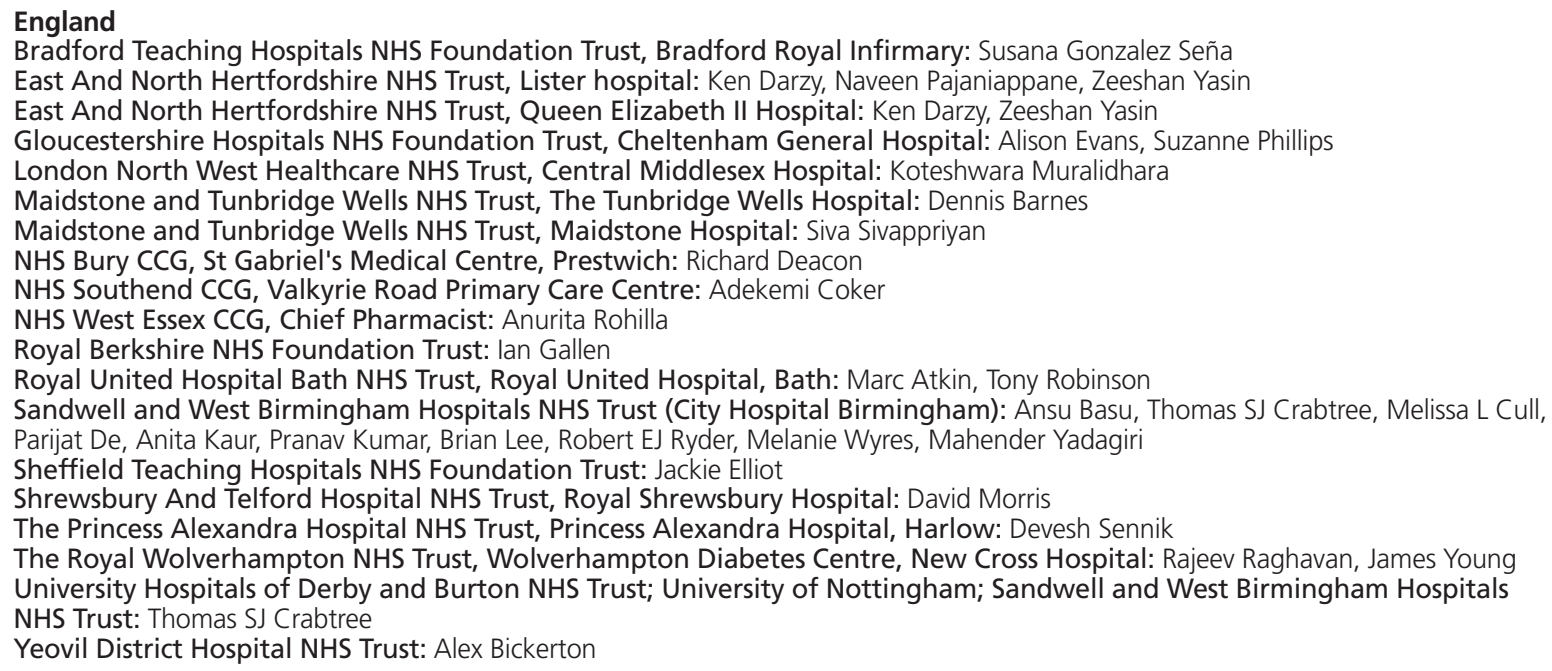

Scotland

West Lothian NHS Trust, St John's Hospital, Livingston: Karen Adamson, Hazel Reid

Wales

Abertawe Bro Morgannwg University NHS Trust: Hatem Eid Abbas Eid, Jeffrey Stephens, David Williams

Acknowledgment

The $A B C D$ nationwide empagliflozin audit is an independent audit supported by an unrestricted grant from Boehringer Ingelheim 